\title{
ROL DEL AGENTE EDUCATIVO EN ACTIVACIÓN DE RUTAS DE ATENCIÓN DE VIOLENCIAS SEXUALES EN LA INFANCIA
}

\section{Role of the educational agent in activating routes of attention to sexual vio- lence in childhood}

\author{
Yusty Carolina Restrepo Segura ${ }^{1} \bullet$ John Jairo García Peña ${ }^{2}$
}

Recibido: 4/5/2017 • Aprobado: 28/6/2017

\begin{abstract}
Resumen
Este articulo es producto de una investigación, cuyo objetivo fue analizar las experiencias personales y profesionales de agentes educativos de primera infancia, en la activación de rutas de violencias sexuales para el restablecimiento de derechos; se concibió desde un enfoque cualitativo, histórico-hermenéutico, con metodología de Estudio de Caso, del programa público Buen Comienzo de Medellín, Colombia, entre 2016 y 2017, para la información generada, se realizaron entrevistas a 10 de sus agentes educativas. Los resultados principales, mostraron inconsistencia en los procesos de formación y cualificación de las agentes educativas, al activar rutas de atención, ante casos identificados de violencias sexuales, en sus espacios pedagógicos. Como conclusión relevante, se evidenció que -ante eventos de vulneración de derechos-, se actúa más por intuición, que por conocimiento profesional, por lo que, los procesos de restablecimiento de derechos se ven obstaculizados, debido a las inconsistencias en la información, al momento de acceder a una ruta de reparación integral.

Palabras clave: derechos del niño; violencias sexuales; agente educativo; cualificación profesional; corresponsabilidad.
\end{abstract}

1. Magíster en Intervenciones Psicosociales, Universidad Católica Luis Amigó. Medellín, Colombia. Correo electrónico: carolinasegura2000@gmail.com ORCID 0000-0003-2565-0416.

2. Magíster en Desarrollo, Tutor tesis de Maestría en Intervenciones Psicosociales. Medellín, Colombia. Correo electrónico: john.garciape@amigo.edu.co ORCID: 0000-0001-8390-5945.
Abstract

This article was product of an investigation whose objective was to analyze the personal and professional experiences of early childhood educational agents, in the activation of sexual violence routes for the restoration of rights; it was conceived from a qualitative, historical-hermeneutical approach, using case study methodology, from the Buen Comienzo public program of Medellín, Colombia, between 2016 and 2017, for the information generated interviews were conducted with 10 of its educational agents. The main results showed inconsistency in the processes of training and qualification of educational agents, when activating care routes, in cases of identified sexual violence, in their pedagogical spaces. As a relevant conclusion, it was evidenced that in face of events of violation of rights, they act more by intuition than by professional knowledge, so that the processes of restoration of rights are hampered, due to the inconsistencies in the information, at the moment of access to an integral reparation route.

Keywords: children's rights; sexual violence; educational agent; professional qualification; co-responsibility. 


\section{Presentación}

La primera infancia cada vez más, es objeto de intervención psicosocial, por ello diversos programas tratan de dar respuesta a sus necesidades, buscando reconocer a los niños como sujetos de derecho y aportando en mitigar su vulnerabilidad, pues los niños, históricamente, han sido objeto de maltrato y violencia en general, donde las violencias sexuales son una de las vulneraciones prevalentes.

El programa Buen Comienzo de la ciudad de Medellín, Colombia, reconocido como política pública, es muestra de dicho accionar de la intervención psicosocial con la infancia, que pretende atender integralmente a los niños, promover su desarrollo, diverso e incluyente desde la gestación hasta los cinco años, desde una perspectiva interdisciplinaria del ciclo vital, desde la protección de los derechos, y desde la articulaciòn institucional (Decreto 01277 de 2013).

Para una sociedad el riesgo de que los niños se encuentren expuestos a diversas formas de vulneración, exige que desde el Estado, se promuevan acciones encaminadas a la atención oportuna de la infancia para restablecer sus derechos.

En Latinoamérica, desde la concepción de Estado de derecho, se da relevancia al Estado y a la familia, como responsables de la seguridad de los niños, de la igualdad de derechos de los miembros de la familia y de la protección integral contra toda forma de vulneración, incluido el abuso sexual; en esa medida, el Estado, la familia y la sociedad, tienen la obligación compartida, de asistir y proteger a los niños.

En este sentido, en Colombia, se cuenta con la ley 1146 del 2007, por medio de la cual se expiden normas para la prevención de la violencia sexual y la atención integral de los niños, niñas y adolescentes abusados sexualmente. Esta ley, estipula explícitamente la obligatoriedad de la denuncia, ante un evento de abuso sexual, responsabilidad que ha de ser asumida por el Estado, la familia y la sociedad como agentes corresponsables.

El código de infancia y adolescencia, ley 1098 de 2006, también resalta el derecho a la integralidad personal de los niños, niñas y adolescentes, como sujetos que deben ser protegidos contra todas las acciones que puedan causarles algún daño, resaltando el derecho a la protección contra toda forma de maltrato y abusos por parte de personas adultas.

Este código también hace mención a las instituciones educativas, como responsables en la detección oportuna, apoyo y orientación en casos de vulneración de derechos, comprometiéndolas con la capacitación del personal educativo, para detectar la vulneración de derechos y realizar la oportuna denuncia ante las autoridades competentes.

\section{La vulnerabilidad de la infancia, un asunto que requiere atención}

En el contexto latinoamericano es evidente la vulnerabilidad social a la que se ven expuestos los niños, por ello ante eventos de vulneración de derechos, la Escuela y muy particularmente el agente educativo, cumplen un papel fundamental en la detección de casos y activación de una ruta para garantizar la atención y restablecimiento oportuno de derechos, sin embargo en estos procesos de atención desde la función de los agentes educativos, se evidencian aspectos que impiden el adecuado restablecimiento de derechos y la atención integral de los niños vulnerados.

En primer lugar, la corresponsabilidad social, el personal que trabaja con la primera infancia, no se encuentra lo suficientemente capacitado para activar y direccionar una ruta ante vulneración de derechos por abuso sexual; sobre lo cual, según Gómez, Salazar y Zuluaga (2011), se evidencia que los docentes de primera infancia, no poseen 
suficientes herramientas técnicas y conocimientos que les permitan hacer una pertinente activación de ruta.

En segundo lugar, es frecuente que los agentes educativos no incluyan a las familias en los procesos pedagógicos para la detección oportuna y subsiguiente atención de las violencias sexuales, Pardo (2009) afirma que, la familia no es consciente de la existencia de las rutas para acudir y exigir el restablecimiento de derechos de sus hijos.

En tercer lugar, los agentes psicosociales están asumiendo la responsabilidad completa, liderando los procesos de garantía de derechos y la activación de rutas, aún teniendo vacíos en sus propios conocimientos sobre el tema; sin embargo, acorde a lo estipulado, desde el programa Buen Comienzo, es función del profesional psicosocial liderar la identificación, registro de rutas para la garantía de derechos, la articulación interinstitucional para garantizar su activación y restablecimiento de derechos (Lineamientos técnicos programa Buen Comienzo, 2015, p. 13, anexo 11).

En cuarto lugar, está la percepción de un deficiente acompañamiento institucional para la denuncia de las violencias sexuales, sobre lo cual aseveran Ramírez, Aranceta y Montoya (2015), que la aplicación de la norma, por los diversos vacíos que presenta, termina vulnerando los derechos fundamentales, así la capacidad de respuesta institucional se ve afectada por un nivel de apropiación insuficiente, que tienen los actores del sistema con respecto a los contenidos de la normatividad en vigencia.

Y como aspecto final, sobre la toma de decisiones respecto a la activación de una ruta, los profesionales involucrados, deben considerar diversas variables, como el apoyo real, que se recibe de las instituciones en las que se ejerce el rol profesional, el tipo de familia a la que pertenece la víctima, el contexto y las características sociales del entorno, la pertinencia de la denuncia y exposición al riesgo personal, lo cual, conlleva a que muchos casos no se pongan en conocimiento de las autoridades judiciales, a veces, por temor, amenazas u otros motivos (Jiménez y Giraldo, 2011).

Lo anterior justifica la pertinencia de esta investigación, cuyo objetivo fue analizar las experiencias personales y profesionales de agentes educativos de primera infancia del programa Buen Comienzo en la activación de rutas de violencias sexuales para el restablecimiento de derechos, de manera que los resultados, permitan proponer espacios de reflexión y de formación, del personal operativo encargado de la infancia.

Este proceso investigativo permitió identificar necesidades y falencias en la activación de rutas de atención integral ante presuntos abusos sexuales, en la búsqueda de que las personas en contacto con la primera infancia, estén lo suficientemente informadas y cualificadas para direccionar a las familias, en estos procesos de restablecimiento de derechos y que las rutas, sean acordes y efectivas para la garantía de una reparación integral.

\section{¿Y qué dicen otros estudios?}

Son diversas las investigaciones que se han hecho referentes a las violencias sexuales en la primera infancia, en relación con la sintomatología asociada a la vulneración, las características familiares de los niños abusados, el perfil de los abusadores, las secuelas psicológicas y emocionales que dejan los abusos en los niños víctimas, los principales agresores y los estratos con mayor índice de abusos sexuales, entre otros.

Sin embargo, el rol de los agentes educativos, en los procesos de activación de rutas y restablecimientos de derechos, es un tema poco estudiado, por lo que los estudios -en término de antecedentes, que de alguna forma hacen mención al respecto- aportaron información relevante.

A nivel internacional, en Chile y en Venezuela, se encontraron investigaciones que revelaron, 
falencias en la formación y conocimiento de los docentes de primera infancia, con respecto a la sexualidad infantil, demostrando que no reconocen su rol en los procesos de vulneración de los niños, como garantes de derechos, a causa del bajo nivel que presentan en conocimientos y habilidades frente a la educación sexual en edad preescolar. Esto evidencia una deficiencia estatal en la formación de los funcionarios públicos. El Estado debe asumir la responsabilidad de generar procesos de cualificación (Gutiérrez, 2011; Navas, 2011).

En el Perú, en Brasil y en Chile, se rastrearon tres investigaciones que muestran que los agentes educativos bien capacitados, tienen mayor eficacia al identificar abusos sexuales, sin embargo, por las desarticulaciones existentes entre programas e instituciones que atienden la primera infancia, estos procesos no se están evidenciando, lo que conlleva a que las intervenciones se ejecuten de forma dispersa y fragmentada (Deza, 2005; Basoalto, 2010; Faria y Albuquerque, 2008).

También en Uruguay y en Venezuela, se analizaron dos investigaciones, que en sus aportes dan cuenta de los roles posibles a asumir, cuando se trata de la protección infantil: el rol de la familia, la escuela y la sociedad, por eso al hablar de vulnerabilidad de la infancia, corresponde asumir en red, familia, Estado y sociedad como agentes encargados de movilizar diversos sectores, servicios, acciones de intervención y entornos protectores (Peroni y Prato, 2012; Morillo, Montero y Colmenares, 2012).

De igual modo, es importante mencionar aportes de investigaciones hechas en Chile, Perú y Argentina, que conciben la prevención, como un trabajo en red, promovido desde la institucionalidad, que se debe dar transdisciplinariamente, mostrando la importancia de la prevención, desde espacios de permanente socialización de los niños, como el hogar, la escuela e instituciones de atención a la infancia (Lefio, Silva y Rivas, 2013; Deza, 2005; Mouescaa, 2015).
A nivel de Colombia, se encontraron investigaciones hechas en Bogotá y en Medellín, que abordan las violencias sexuales, haciendo énfasis en el rol de la escuela, en aras de garantizar el desarrollo pleno e incluyente de la niñez, destacando que existe una necesidad de articular la institucionalidad a nivel público y privado, para la garantía del desarrollo integral de la infancia y el trabajo interdisciplinario de los profesionales, que están en interacción con los niños (Rodríguez, 2003; Jiménez y Giraldo, 2011; Sierra, Arboleda y Agudelo, 2011).

En Bogotá y en Bucaramanga, Colombia, se investigó sobre los procesos de atención oportuna, ante las denuncias recibidas por las instituciones, responsables de la recepción y atención, para la restitución de derechos ante eventos de violencia sexual. Muestran que se presentan falencias, como la repetición de diligencias por parte de la familia víctima, que conlleva a múltiples declaraciones y exploraciones de parte de los funcionarios públicos, vacíos en la ruta de atención, ineficacia en la prioridad de recibir las denuncias, falta de articulación entre las entidades responsables de la atención. Todo esto, revela la necesidad de generar espacios de reflexión y conciencia frente al rol protector como funcionarios, la oportunidad de la ejecución de una ruta disponible para la restitución de derechos y la relevancia de generar compromisos con escuelas, hospitales y fiscalías, como redes secundarias, que propicien la denuncia, prevención y judicialización (Sánchez, 2008; Galeano, 2012; Pardo, 2009).

Por último, de manera puntual, Medellín es pionera -en Colombia- en atención a la primera infancia, y la preocupación por su protección integral, ha llevado a la gestión de acciones en investigación sobre la vulneración de los derechos de los niños.

En este sentido, algunos autores se han preguntado sobre los roles que desempeńa un adulto significativo para proteger a los nińos contra toda forma de maltrato, dando paso al nombramiento del agente 
educativo, la familia, la sociedad y el Estado, como actores corresponsables en la protección integral de la niñez. Estas investigaciones, demuestran que la formación de docentes y las estrategias que utilizan para detectar el abuso sexual en edad preescolar, es insuficiente, que no cuentan con la información, los conocimientos teóricos, prácticos e instrumentos necesarios para realizar una oportuna detección de abusos sexuales, siendo su herramienta más útil la intuición guiada, lo que muestra la necesidad de sensibilizar y formar a los profesionales que atienden la primera infancia en la atención primaria, en detección y prevención del maltrato infantil (Gómez, Salazar y Zuluaga, 2011; Zapata y Ceballos, 2010).

Otras investigaciones similares, resaltan la importancia de las acciones mancomunadas para la garantía de protección de la primera infancia, resaltando el trabajo articulado en redes de apoyo, entre el sector público y el privado; así, la garantía del cuidado y los derechos de los nińos es un asunto público, que ha de ser apoyado, creando espacios de reflexión y ambientes que garanticen su protección, generando el compromiso político, gubernamental y legislativo, así como cambios en la comunidad y la función de la familia, como agentes corresponsables de la prevención y recuperación ante eventos de abuso sexual (Murillo, Gómez y Quiroz, 2011; Villegas, Zapata y Vega, 2011).

\section{Metodología}

Esta investigación se orientó desde el paradigma histórico hermenéutico, con un enfoque cualitativo, el cual, según Dilthey (1990), hace posible comprender e interpretar los discursos, en este caso particular, de las agentes educativas del programa Buen Comienzo de la ciudad de Medellín, Colombia, en relación con el conocimiento que tienen, desde su propia experiencia laboral y personal, en la activación de rutas ante eventos de violencias sexuales en la infancia. Interesaba reconocer sus experiencias personales, vivencias, percepciones, significados, conocimientos teóricos y concepciones frente a esta temática.

Desde este enfoque, la experiencia humana cobra sentido en el contexto donde se interactúa, como un acto comunicativo fundamental. Por lo tanto, no es posible descontextualizarla y siempre es factible abordarla desde la cotidianidad que las situaciones implican (Bourdieu citado en López, 2001, p. 33).

En la misma línea, la estrategia metodológica propuesta fue el Estudio de Caso, que según Creswell (2013), trata de comprender, desde la perspectiva de los participantes, aquello que los caracteriza particularmente, pero también lo que los hace comunes. En este sentido, el programa Buen Comienzo de la ciudad de Medellín, es el caso estudiado.

Es necesario comprender, desde la experiencia de sus agentes educativas, lo que expresan como ejercicio de su hacer, no desde una actitud meramente interpretativa, que busca la coherencia interna del texto analizado, sino desde el respeto pleno por el relato del hablante, es decir, estas experiencias se configuran desde las vivencias que el ejercicio laboral del mismo programa posibilita (López, 2001).

Se empleó, como técnica de generación de la información, la entrevista semiestructurada, a partir de un guión que constó de 40 preguntas, construidas desde las categorías de análisis, que con base en los referentes conceptuales y la pesquisa de los antecedentes, guiaron esta investigación. Este instrumento, tuvo un criterio de validación, mediante un grupo focal con profesionales expertos de la alcaldía de Medellín.

La entrevista se realizó a diez (10) agentes educativas, que desempeñan diferentes roles dentro del programa Buen Comienzo, fue fundamental la escucha atenta, de cómo han vivido su ejercicio profesional con la infancia, buscando reconocer cada 
esencia de comprensión personal, desde su hacer laboral cotidiano, que las expone y compromete continuamente.

En el momento de elección de las participantes, se identificaron cinco (5) entidades prestadoras del servicio a la primera infancia, que operan a nombre del programa Buen Comienzo, se seleccionaron dos (2) participantes de cada una, los criterios de elección, consistieron en que fueran agentes educativas del programa y que llevaran mínimo dos (2) años de experiencia con primera infancia. Las diez (10) entrevistas fueron grabadas, transcritas y almacenadas en medio electrónico. Para la codificación y análisis de la información se usó el programa Atlas.ti.

\section{Resultados}

Se presentan con base en la categorización, realizada a partir del referente conceptual y el proceso de escucha a las participantes, cada categoría de análisis detalla sus aportes, desde sus decires, reflejando sus experiencias profesionales y personales, sobre el estudio temático.

Las categorías definidas fueron: la experiencia (personal y profesional), la cualificación profesional, el desarrollo humano, las violencias sexuales, la protección integral, las rutas de atención y la corresponsabilidad, categorías que se desarrollan a continuación.

\section{Experiencias profesionales y personales}

Como categoría central de esta investigación, hace referencia a los conocimientos adquiridos y construidos por las agentes educativas desde su hacer profesional sobre las violencias sexuales.

En el caso específico del programa Buen Comienzo, la experiencia se convierte en un factor fundamental, que determina el proceder de las agentes educativas, ante situaciones de vulneración de derechos de los niños. Ante este hecho, se evidenció que aquellas agentes educativas que se habían enfrentado, en su experiencia profesional, con situaciones de violencias sexuales, mostraban mayor seguridad al dar respuesta, frente a los procesos de activación de rutas para el restablecimiento de derechos, fundamentando su respuesta en esos procesos vivenciados.

En cambio, otras agentes educativas, que no habían experimentado aún en su ejercicio laboral, este tipo de situaciones, tendieron a dar respuestas desde su sentir y su creer, más que desde un conocimiento adquirido e incorporado en su formación profesional.

Frente a estas dos posturas, pueden ilustrarse los siguientes aportes. Las agentes educativas que no se han enfrentado a este tipo de situación:

"Yo creo que uno nunca va a estar preparado para eso, o por lo menos como todavía no me ha tocado, ojalá y no, pero yo creo que psicológicamente es muy dificil aceptar eso, bueno aunque estamos en una sociedad en la que eso se ha convertido como tan normal...". (E.4, p. 49)

Las agentes educativas que sí se han enfrentado a este tipo de situación:

Sí, me ha tocado vivenciar abuso sexual, maltrato y negligencia en el cuidado, y se ha posibilitado desde mi rol como agente educativo, activar de alguna manera la ruta más cercana. Para el caso de abuso sexual como tal, el procedimiento-inicialmente- fue el contacto con la familia, para indagar y saber acerca del tema, porque en el momento no teníamos el acceso a esa parte de acompañamiento psicológico que nos pudiera favorecer eso, entonces se hizo directamente como agente educativo docente... (E.8, p. 2).

Por tanto, se observa que la experiencia laboral, da más elementos de acción, es por ello que los 
profesionales del área psicosocial son referentes y, a nivel del programa, asumen más responsabilidad sobre el ejercicio de la Ruta, que les exige constantemente, fortalecerse para tener argumentos conceptuales, desde su formación profesional, en el área de protección. Mientras que los otros profesionales -como las coordinadoras, nutricionistas, docentes y auxiliares pedagógicas-, se apoyan más en la experiencia del hacer vivencial y de la capacitación recibida dentro del programa. Por lo que es común que fusionen información y que, en ocasiones, contradiga la normatividad que exige corresponsabilidad por parte de todos los que tienen a su encargo la infancia. Un aporte que lo sustenta:

Los agentes educativos no están preparados, porque es como te decía, o sea, va más allá de que ellas tengan información, porque ellas fácilmente te pueden decir a ti cuál sería el paso a paso a seguir, en el momento que hagan la detección de un abuso sexual, pero de ahi a que ellas lo realicen... no lo hacen, por miedo, porque no se sienten capaces, porque ya la responsabilidad está delegada sobre el psicosocial, entonces desde la propia entidad, le recae la carga al agente psicosocial (E.10, p. 41).

Con respecto a la preparación de las agentes educativas para hacer frente a las diferentes situaciones de vulneración de derechos, en el programa Buen Comienzo se encuentra estipulado que su personal operativo debe estar cualificado para prestar la atención integral a la primera infancia, las instituciones prestadoras del servicio han de propiciar esos espacios educativos para nutrir los conocimientos y el quehacer de sus profesionales.

\section{Cualificación profesional}

Sobre este aspecto, desde este programa público, el componente de gestión del talento humano es responsabilidad institucional, en términos de brindar cualificación permanente al personal operativo, con miras a garantizar una atención integral a la infancia, formación que ha de surgir desde la caracterización de las necesidades y expectativas de los educadores; pero este componente tan importante, se deja a la voluntad de cada profesional. El siguiente aporte respalda esto:

Bueno se ha participado en capacitaciones de las que hizo Buen Comienzo el año anterior, que -de alguna manera-lo hicieron por ciclos $y$ se hizo sobre la vulneración de derechos en general, no como formación a nivel del abuso sexual como tal, yo creo que eso sigue siendo un tabú, $y$ de hecho no lo programan tanto, es como menos cercano, yo participé a nivel, ya personal, en un proceso de formación al respecto, y lo bice con otra entidad que ha atendido mucho niños con vulneración en abuso sexual, no con Buen Comienzo, pero básicamente lo hice por interés personal, cuando se nos presentó un caso de abuso sexual... (E.8, p. 45).

Aunque es claro desde los lineamientos institucionales, la obligatoriedad de garantizar la preparación oportuna de los agentes educativos sobre la atención integral de la infancia, en las verbalizaciones de las agentes educativas, no se encontró coherencia entre lo que manifiestan conocer y lo definido por el programa en sus lineamientos técnicos.

En esta medida, las agentes educativas, perciben que el proceso de capacitación ofrecido presenta falencias, con respecto al conocimiento que les queda, las metodologías utilizadas y sobre todo, en el acompañamiento, por parte del programa; lo cual, puede generar como resultado, poca sensibilización y apropiación, por parte de ellas, en los temas expuestos en los planes institucionales. Este aporte lo sustenta:

A ver... yo qué le digo, que me gustaría aprender más sobre el tema, me gustaría que conociéramos, de verdad, todas esas rutas que considero son tan importantes, y no me habia dado cuenta hasta esta entrevista, porque yo me siento muy ignorante en este sentido, no sé qué hacer, no sé qué es la ruta, o qué hacer con eso, cómo involucrarme 
ahi, y yo siento que - de pronto-deben de tener un conocimiento más las docentes, no sé cómo auxiliar, siempre nos encargamos de otras cosas, no sé cómo hacerlo (E.9, p. 46).

En estos procesos de cualificación, el compromiso sigue siendo delegado al profesional psicosocial, quien lidera los procesos de prevención, atención y denuncia de las violencias sexuales que se presenten en la infancia, pero esto no puede ser la excusa para que el resto del equipo interdisciplinario evada la responsabilidad conjunta de una atención integral, que exige cualificación de todos sus integrantes, desde su diferentes roles.

Según la Política Pública de Primera Infancia, en sus lineamientos se manifiesta que este es un asunto de corresponsabilidad y construcción conjunta en los equipos interdisciplinarios. $\mathrm{Al}$ respecto:

Yo creo que, generalmente, ni siquiera las mismas cabezas de las entidades tienen claro el proceso, es decir, lo hacen y se delega al psicosocial. Cuando este profesional falta, es más caótico tomar la decisión y, tristemente, yo creería que Buen Comienzo deja al psicosocial solo, él asume, muchas veces, activa la ruta y pone en riesgo su vida, sin el apoyo suficiente del Programa, que es quien tendria que velar y promover que estas rutas se den (E.8, p. 12).

Esto implica déficit en la atención integral, lo cual, lleva a pensar la intervención, en pro del desarrollo integral de los niños, las familias y la comunidad en general. Pues mientras haya debilidades en la cualificación profesional de las agentes educativas, en un programa público, es más difícil superar las problemáticas que atentan contra el óptimo desarrollo de la infancia.

\section{Desarrollo Humano}

Para garantizar la atención integral a la infancia desde el programa Buen Comienzo, se toma en consideración el desarrollo humano y social como un factor protector para el beneficio de los niños, lo cual permite entender el desarrollo desde una mirada integral, que permite un acompañamiento oportuno, promoviendo condiciones para la calidad de vida.

Los aportes de las agentes educativas mostraron que se promueve el desarrollo humano empleando diferentes estrategias pedagógicas, llevadas a cabo desde los espacios significativos, por medio de planeaciones educativas, según las necesidades e intereses de los niños, empoderándoles en su proceso de formación y protección desde el autocuidado. Aportes al respecto:

"Nosotros allá, por ejemplo, sí hacemos sobre el
autocuidado, entonces ahi ya se les dice quién
debe tocarlos, quién no, donde se dan los besitos
y todas esas cosas, para que ellos sepan cómo se
debe hacer, lo trabajamos por medio de videos,
imágenes, cuentos, obras de teatro"(E.1, p. 32). Por otro lado, profesionales como las agentes educativas psicosociales y las coordinadoras pedagógicas mostraron mayor seguridad y conocimiento frente a estrategias directamente dirigidas a la prevención de las violencias sexuales, llamando las cosas por su nombre, sin evadir el tema. El siguiente aporte lo expresa:

Bueno, la prevención de las violencias sexuales se puede hacer a través del juego, a través de las respuestas claras y pertinentes a los niños, cuando hacen interrogantes frente a sus inquietudes sexuales, frente a esos lenguajes que ellos establecen cuando cuentan sus historias, hay que estar con una escucha atenta, no es jugar por jugar, se trata de estar atentos a esos juegos y a esos diálogos que establecen, qué significan, es posibilitarles el acceso a la información que - de alguna manera-el mismo medio ya les provee, que si es el video, que si es la canción, que si es la posibilidad de imágenes, los cuentos... hay literatura que ya favorece ese acercamiento, entonces creo 
que son esos espacios que se pueden dar en los momentos pedagógicos y hay que aprovecharlos (E.8, p. 24).

Para garantizar la formación y desarrollo humano de la infancia en las comunidades, el programa cuenta con planes institucionales, para la educación y formación a agentes educativos, familias, personas significativas y comunidad. En este punto, es importante resaltar que, si bien se han emprendido diversos esfuerzos de capacitación y formación, también se ven falencias, hay dificultades para convocar al 100\% de las familias, al igual que para concretar los encuentros que a veces se ven afectados por las dinámicas mismas del programa.

Con las familias, el tema de la vulneración de derechos, igual que como pasa con las agentes educativas, se trabaja solo una vez al ańo, en encuentros que no logran la participación total de todas las familias atendidas. Este aporte lo demuestra:

Generalmente siempre se hace un encuentro educativo al año, que es exclusivamente de la activación de rutas y la prevención de violencia, entonces el encuentro educativo, aunque a veces es muy complicado porque trabajamos con bebés muy pequeños, siempre se trata de que las familias reconozcan todos estos tipos de signos y sintomas en casos de abusos o algún tipo de violencia, qué rutas pueden activar, cómo las pueden activar, porque a veces ellos tampoco quieren contarnos lo que sucede (E.7, Psi1. p. 13).

Los lineamientos técnicos del programa, contemplan aquellos contextos que pueden afectar directa e indirectamente el desarrollo de los niños, como son la familia y los entornos educativos, sobre los que, por tanto, tendría que intervenirse de una forma más consistente por parte de la institucionalidad.

Sin embargo, es evidente que desde la implementación del programa, se falla en los procesos de fortalecimiento tanto familiar, como educativo, lo cual no está permitiendo - de forma cabal- fortalecer el desarrollo adecuado de los niños para una protección integral, en términos de garantías del desarrollo humano, al que tiene derecho la infancia.

\section{Protección integral}

Buen Comienzo plantea la protección integral y la inclusión, como acciones relevantes para la garantía del desarrollo infantil, generando capital social, donde la protección, sería el principio rector y eje articulador, mediante el cual se hace posible reconocer a los niños como sujetos de derecho, para satisfacer sus necesidades y desarrollar sus potencialidades (Lineamientos técnicos para la prestación del servicio de atención integral a la primera infancia, 2015).

Desde esta concepción se promueve el cuidado desde la integralidad emocional, social y física de cada niño.

En esta vía, la protección integral se aborda, en primer lugar, con respecto a la prevención de las violencias sexuales por medio de estrategias pedagógicas y procesos formativos con familias, agentes educativos y niños, en búsqueda de empoderar a cada uno de estos grupos poblacionales, desde sus diferentes roles, en el compromiso con la infancia. $\mathrm{Al}$ respecto, las agentes educativas mencionan lo siguiente.

Yo pienso que es fundamental... los espacios pedagógicos son sumamente importantes, porque los padres de familia, los niños y hasta nosotros mismos, adquirimos - de estos procesos- información que no tenemos por ningún otro lado, que siempre necesitamos recordarla de algún modo; pero qué bueno que fuera no solamente hacerlo como una manera de taller, sino dejar siempre como un registro en alguna parte, que yo siempre lo viera y recuerde que eso está por ahi (E.3, Nu1). 
En segundo lugar, desde la confidencialidad definida por el programa, que incluye el manejo de la documentación, y de la información personal sobre la atención y acompańamiento psicosocial. Este asunto aunque se encuentra claramente estipulado, a partir de un componente de protección, sin embargo no ha sido interiorizado por las agentes educativas, y en alguno casos donde ha existido vulneración de derechos existe dificultad en el manejo confidencial de la información.

Eso es otro tema delicado, porque primero uno no puede estar divulgando que el niño fue abusado sexualmente, entonces obviamente eso solamente lo sabe la docente que lo detectó, supuestamente, la psicóloga y la coordinadora, pero a veces eso tampoco lo saben manejar dentro de las instituciones, porque entonces la docente lo detecta y lo primero que hace ahi es: ve, yo te cuento, imaginate que este niño..., entonces toda la institución se dio cuenta de que ese niño fue abusado, entonces ya lo empezamos a mirar con lástima, con pesar; entonces, desde ahi también hay que hacer toda una sensibilización con los agentes educativos, de que ese tipo de cosas no se divulgan, o sea eso es muy personal del niño y de las familias, entonces muchas veces lo están revictimizando de esa manera, cuando empezamos a mirarlo como desde ahi. Entonces, sí, siento que falta mucho en las instituciones. Más: eso se llama respetar la privacidad de ese niño (E.2, Co1).

En tercer lugar, también es importante analizar la protección, desde el punto de vista del restablecimiento de derechos, que abarca las acciones y gestiones integrales para restaurar con plenitud y efectividad los derechos vulnerados. El Estado, representado en el programa Buen Comienzo y sus instituciones prestadoras del servicio de atención integral, deben asumir y liderar las acciones administrativas para desarrollar el debido restablecimiento de derechos. A pesar de ello, las percepciones de las agentes educativas mostraron que, tanto las instituciones, como la dirección del Programa, no acompañan activamente estos procesos de restablecimiento de derechos. Sobre este asunto:

Si la agente educativa no sabe, realmente, identificar o sospechar de este tipo de casos, siempre debe hacer una remisión al psico inmediatamente. Y algo que pasa, y que también he visto, es que si los agentes educativos no saben cómo manejar el abuso, esperan hasta que llegue el psico; en un trabajo anterior, me tocó que no habia psico, hasta que yo llegué, llevaban dos meses sin psicosocial y llevaba tres meses, un caso de abuso, que ni siquiera habia sido denunciado, la coordinadora pedagógica también tenía conocimiento y nadie habia hecho nada, ningún tipo de acompañamiento ni de sensibilización, solo porque estaba la vacante, entonces yo considero que siempre tiene que haber alguien pertinente, o sea asi no sea un psico de planta o si está la vacante... alguien... alguien tiene que responder por eso (E.7, Ps1).

De esta forma, para garantizar la protección integral, se busca la detección oportuna de las violencias sexuales y la activación de ruta, como un asunto que requiere de un acompañamiento, por parte de los profesionales que lideran el proceso, especialmente los agentes psicosociales, cuestión que invita a profundizar en la siguiente categoría.

\section{Rutas de atención}

En este aspecto se pudo identificar que se delega a los equipos interdisciplinarios, la construcción colectiva de las rutas y la divulgación de las mismas, sin embargo, no hay una sincronización, un lenguaje institucional, entre los diferentes profesionales para su construcción.

A este respecto, las agentes educativas mostraron diferencias significativas en la activación de rutas 
para el restablecimiento de derechos, por ello-para algunas agentes educativas- la ruta parece efectiva $y$, para otras, no. Lo cual puede valorarse como poco eficiente -y hasta peligroso- en un programa público, que tiene por misión la protección integral y el desarrollo humano de sus niños; aspectos que se lograrían, si existiera unicidad y pertinencia en la ruta de atención, conllevando a efectividad institucional.

Para dar un ejemplo en las formas de proceder en la activación de rutas, una agente educativa refiere:

En la sede donde nosotros estamos, lo que se hace es que primero se llama a la psicóloga para que ella sepa la situación, ya ella dice hacia donde debemos movernos, casi siempre es Caivas, se tiene que hacer la conexión con la familia para poderle avisar que hubo un abuso sexual, empezar a determinar ¿por qué?, ¿quién es?, la Policía de Infancia y Adolescencia también interviene ahi (E.3, Nu1).

Otra agente educativa expresa diferencias en este proceso de activación de rutas:

Primero hay una observación del agente educativo, que es el que está más en contacto con el niño, luego se habla con el adulto responsable del niño para indagar más acerca del comportamiento, luego se le comenta a la psicosocial (pues ellas también tienen sus procesos), que miran desde su área .. y ya, dependiendo como de la situación, cómo van a remitir ese caso de abuso, a qué profesional, o si requiere que le hagan el examen que requiere el niño y su respectiva demanda (E.2, Do2).

Es de resaltar, que las inconsistencias que se identificaron, comprometen el bienestar y la protección de las víctimas en el proceso de restablecimiento de sus derechos.

Así, en las participantes apareció, reiteradamente, que la familia es la única responsable de llevar a cabo un proceso de denuncia y, si estas se niegan a denunciar, la atención integral se interrumpe, persistiendo la vulneración. Sobre lo cual hay un error perseverante en las agentes educativas, porque en todos los niveles -desde docentes a coordinadorastienen interiorizado que esa decisión familiar, de no denunciar, se respeta, sin que ellas puedan hacer algo al respecto.

Ese aspecto es contrario a lo establecido por la ley 1146 de 2007, que en el artículo 12 menciona la responsabilidad del agente educativo $-y$, en general, de toda persona adulta conocedora- y la obligatoriedad de la denuncia de cualquier violencia sexual, ante autoridades judiciales para dar inicio al proceso de restablecimiento de derechos de los niños víctimas.

El siguiente aporte muestra las fallas que se presentan en la activación de una ruta, a partir de los conocimientos que están manejando las agentes educativas:

Pero, entonces, el problema abi es que, cuando ya tocamos la violencia sexual, esos temas familiares, la familia como tal, lo que hace es negarlo, entonces a veces la ruta se queda ahi, porque la idea sería que a ellos se les dan las indicaciones de que vayan al Caivas, más que a la fiscalia y, a partir de ahi, empezar a hacer esa restitución de derechos, pero las familias, como son personas tan cercanas, no lo hacen, entonces -en los casos que tuvimos- una de las familias, la mamá prefirió sacar al niño de la institución y la otra mamá pues, echó al hijo de la casa, pero va hasta ahi, no hay una restitución de derechos como tal. Es muy dificil, uno llega hasta un punto, la coordinadora y la psicóloga se sientan con esa familia, después de que ya haya pasado el proceso de evaluación del niño y la familia para que hagan esa denuncia, pero uno no los puede obligar, entonces hay muchos que sí la hacen, y ahi se va acompañando y se llaman a ver cómo les fue, ¿cierto?, y todo el cuento, y se 
va haciendo el acompañamiento como tal, pero las familias que no, hay muchas que antes sacan los niños de los hogares infantiles, cuando uno hace, entre comillas, ese tipo de descubrimiento y eso hay que respetarlo (E.2, Co1).

Otro aspecto a resaltar, sobre el uso de las rutas, es que -desde Buen Comienzo- se busca mejorar sus procesos de activación. Así, los equipos interdisciplinarios implementan acciones de articulación con entidades externas que benefician la formación a familias y agentes educativas en violencias sexuales y, así mismo, permiten que la activación de la Ruta se dé oportunamente, apoyados en estas entidades para la recepción de denuncias y la atención de los niños vulnerados.

Sobre este asunto una agente educativa verbaliza:

"Casi siempre está la Policía de Infancia y Adolescencia que es la que más se articula, también nosotras desde el área de cada una, tenemos contactos en ICBF que también, de una $u$ otra manera pueden influir pues, como en tomar una decisión con un niño o recibir un tipo de apoyo para ver qué proceso a seguir con ellos"(E.3, Nu1).

Sin embargo, si bien se cuentan con articulaciones, en el momento de detectar un presunto abuso sexual, aún no se logran atenciones oportunas y de restablecimiento inmediato de derechos. Las rupturas presentes en estos procesos siguen siendo significativas, por lo que la revictimización se convierte, en un asunto de preocupación en el trabajo de lo público, parece que, al final, nadie asume desde sus funciones respectivas una responsabilidad que -en el contexto actual-, se exige como una acción compartida que se denomina corresponsabilidad.

\section{Corresponsabilidad}

$\mathrm{Al}$ indagar por la corresponsabilidad que tienen las agentes educativas frente a la protección de un niño que ha sido vulnerado, entienden que consiste en la protección a los niños, como una garantía de que no se repita la vulneración y se dé el acompañamiento desde las instituciones, al niño y a su familia.

Esto indica que, de forma general, hay conciencia de la corresponsabilidad con respecto a la infancia, pero a causa de las falencias identificadas en su formación y cualificación profesional, la corresponsabilidad se queda como un asunto retórico, que difícilmente pasa del papel y, del decir, a la práctica en el diario compartir con los niños.

Sobre este asunto:

Inicialmente todo el equipo interdisciplinario debería conocer las rutas de atención, todos deberian estar comprometidos y ser correspondientes, perdón corresponsables, de la situación, y no solamente que sea el psicosocial la persona encargada de llevar todo el proceso y la activación de Ruta, porque en muchas ocasiones las docentes detectan lo que está pasando y se guardan la información por miedo o por no saber cuáles serían (sic) el procedimiento adecuado a realizar y esperan hasta que llegue un agente psicosocial y, cuando las entidades no tienen psicosocial, pues hasta ahi llega el apoyo, y se quedan con la información hasta que alguien les dé alguna luz para ver qué hacer. Entonces, antes de empezar mi trabajo como agente educativa o como psicóloga, como nutricionista, yo debería tener información acerca de cómo puedo identificar cuando a un niño se le está vulnerando el derecho, cuál es mi papel, qué rutas activar, qué apoyo institucional tener, qué red social, ¿cierto? y más si, por ejemplo, están sufriendo violencias sexuales, pues teniendo en cuenta que nosotros, los psicosociales, somos los competentes para manejar las situaciones, pero ellos, el resto del equipo, también podrian y tendrian que aportar (sic) (E.10, PS2).

De tal forma que también se hace necesario resaltar un factor importante que interfiere en los 
procesos de la corresponsabilidad, el miedo a represalias.

En los discursos de las agentes educativas apareció constantemente el miedo a denunciar por múltiples características de los entornos, las familias y las personas implicadas en los casos de abuso sexual detectados, por lo que su responsabilidad civil de denunciar, cuando no hay una respuesta positiva de la familia para iniciar el proceso de restitución de derechos, se muestra invisibilizada y se omite, al momento de preguntarse por su seguridad, su vida e incluso, por conservar su trabajo.

Muestra de ello, la siguiente contribución:

El tema de las violencias sexuales es muy dificil, porque es uno meterse al campo de alguna manera de las personas que son -entre comillas- malas. Porque, por ejemplo, uno - como agente educativo - activa la Ruta, entonces ya se le viene la familia encima, entonces ya se vienen las amenazas, ya uno se tiene que ir del barrio o tiene que dejar su trabajo, entonces es un tema muy dificil, pero por lo menos debería haber más capacitación (E.4, Do2).

\section{Discusión}

Aunque en Colombia y, especialmente en Medellín, se ha hecho una gran apuesta por la atención y protección integral a la primera infancia, por medio de políticas públicas que nombran los nińos como sujetos de derecho, es evidente que falta mayor compromiso y sensibilización de los funcionarios que conforman la institucionalidad, los equipos educativos e incluso, las mismas comunidades y familias. Como se ha identificado en esta investigación, estas entidades no se encuentran lo suficientemente sensibilizados y comprometidos con su rol corresponsable en la protección de la infancia.
Si bien existe una normatividad y unos lineamientos coherentes en el papel, que regulan el funcionamiento de los programas públicos sobre la infancia, en su ejecución se presentan brechas que comprometen su objetivo superior de garantizar entornos protectores e incluyentes para los nińos.

La protección integral de la infancia, además de ser una responsabilidad política asumida por el Estado, es una responsabilidad civil que involucra a todas las personas pertenecientes a una comunidad. La familia, la escuela y otras instituciones de la red social, como los agentes educativos y la sociedad en general, están llamados a reflexionar en sus roles, como agentes corresponsables para la garantía del desarrollo humano global de los niños.

El rol del agente educativo, como se ha definido desde los mismos antecedentes de este estudio, es necesario en los procesos sociales para la creación de comunidades protectoras para los nińos, quien tiene que asumir, más allá de un rol educativo, un rol protector.

Como agente protector, sería posible generar conocimientos, habilidades y saberes que permitan crear entornos seguros de protección, a partir de los cuales sería posible identificar oportunamente la vulnerabilidad y activar adecuadamente y con pertinencia, una ruta de restablecimiento de derechos cuando se requiera, optimizando tiempos y evitando la revictimización en los procesos de atención, desde la misma detección, garantizando así una oportunidad.

En esta vía, se retoman a continuación las categorías de análisis, para mostrar cómo, en cruce con los antecedentes y los conceptos referenciados, estos hallazgos hicieron posible responder a la pregunta guía de investigación.

\section{Experiencias profesionales y personales}

Para Tamir (2005), existen dos formas de adquirir el conocimiento, una como profesional, referente 
a los conocimientos y habilidades necesarias para tener éxito en una profesión y obtener el consentimiento de la comunidad profesional, y la otra, es el conocimiento práctico personal, el cual engloba la experiencia y permite hablar como una persona instruida e informada.

Se trata de un conocimiento que se descubre en las acciones de las personas, por ello la conducta de una persona en su campo profesional termina siendo el resultado de la interacción entre el conocimiento profesional y el personal, el conocimiento es adquirido por medio de las experiencias dirigidas, proyectadas y planificadas, al igual que la totalidad de las experiencias de vida encontradas en cada persona. (Tamir, 2005)

En este sentido, esta investigación evidencia, en relación con los trabajos realizados por Gutiérrez (2011) y Navas (2011), que la débil preparación profesional y personal de los agentes educativos para hacer frente a una situación de vulneración de derechos en sus espacios pedagógicos, referencia más su propia experiencia.

Las agentes educativas de Buen Comienzo refieren con frecuencia experiencias, que indican vacíos significativos en los conocimientos teóricos y prácticos frente a las violencias sexuales y vulneración de derechos en general. Asunto que revela rupturas significativas en los procesos de atención y activación de rutas para el restablecimiento inmediato de derechos en la infancia.

Hay debilidades en la preparación individual e institucional que -como funcionarias educativas- reciben en su formación. Esto conlleva a que procedan, en sus acciones de atención y detección, más desde experiencias previas e intuición, que desde su preparación laboral; resultado que concuerda con lo mencionado por Eliana Gómez, Laura Salazar y Catherine Zuluaga (2011), quienes sostienen que las agentes educativas usan la intuición guiada para identificar e intervenir los abusos sexuales, y esto se debe a las falencias que tienen en conocimientos teóricos y prácticos relacionados con el tema.

Las experiencias, en general, son las guías de sus comportamientos y maneras de proceder ante un caso de vulneración de derechos y, ante la escasez de estas experiencias en el campo profesional y personal, se presentan múltiples vacíos en el manejo y en el proceder de activación de rutas, lo que conlleva a la revictimización de los niños, hecho relacionado con la poca cualificación promovida desde la misma institucionalidad.

\section{Cualificación profesional}

La cualificación profesional es una consecuencia de la formación del trabajador como resultado de su propio bagaje intelectual y compromiso personal, junto con aquella formación, que requiere el puesto de trabajo donde se desarrollan las funciones. En este proceso, intervienen las capacidades que las tareas exigen y, por otro lado, las características de cada uno de los puestos, definidas por una estructura laboral. Así, una persona se encuentra cualificada cuando el desempeño laboral que tiene, le permite obtener los resultados esperados con los recursos y el nivel de calidad (Olaz, 2011).

Como lo resaltan investigaciones consultadas, el Estado es quien debe asumir la responsabilidad de generar procesos de cualificación a agentes educativos para responder frente a la protección efectiva de la vida e integralidad de la infancia, pero también, de los adultos que denuncian, y así garantizar el restablecimiento de derechos (Basoalto, 2010; Peroni y Prato, 2012; Deza, 2005).

Los docentes de educación inicial, están llamados a conocer y analizar las diferentes situaciones que ponen en riesgo a los nińos, y tomar medidas frente a la vulnerabilidad percibida, a partir de promover una comunicación que permita, de forma conjunta, 
institucionalidad y familia, tomar decisiones propicias a las necesidades de los niños.

Desde esta perspectiva, la cualificación de un profesional depende, además de su propio interés y voluntad, de la formación promovida desde las instituciones; con este estudio, fue posible percatarse que las falencias identificadas en este aspecto, van ligadas a la falta de espacios de formación, reflexión y análisis que permita a los funcionarios públicos tomar conciencia de su corresponsabilidad en los procesos de restablecimiento de derechos y garantía de protección integral de la infancia.

Esto también lo había identificado, Evelyn Gutiérrez (2011), quien afirma que existen representaciones sobre la infancia que demarcan el actuar de las educadoras frente a la infancia, es decir, que es notable que desconocen principalmente el concepto de garante de derechos de la infancia. Además, muchos de los funcionarios públicos que ejercen un rol en el Estado, también lo desconocen.

El Estado está llamado a generar procesos de formación, información y reconocimiento que les permita a los agentes educativos identificarse como garantes de derecho. A manera de reflexión, queda la necesidad de abordar los derechos de los niños por medio de la transversalización de las prácticas educativas con un enfoque de derechos.

\section{Desarrollo Humano}

La propuesta para garantizar la atención integral de la primera infancia desde el programa Buen Comienzo, toma en consideración teorías integrales e integradoras, que pretenden apuntar a los derechos, al desarrollo humano y social de los niños. En este sentido, es oportuno traer a colación la propuesta teórica del Desarrollo a Escala Humana de Max-Neef (1994), la cual, desde una perspectiva de la satisfacción de las necesidades humanas fundamentales, la autodependencia y las articulaciones orgánicas, insiste en que el protagonismo de las personas, ha de ser real, logrando la transformación de la persona-objeto en persona-sujeto.

El concepto de ecología del desarrollo humano de Bronfenbrenner (1994), también resalta la importancia de los entornos naturales y sociales en los que ocurren las vivencias y experiencias que le dan sentido al desarrollo de los niños. Además, el desarrollo de las libertades y capacidades desde Amartya Sen (2000), propone que el ser humano es medio y fin del cual depende el desarrollo de una sociedad que debe garantizar las oportunidades para el desarrollo de las capacidades de las personas, en esta medida, la libertad es el fin principal del desarrollo humano.

Teniendo en cuenta estas acepciones teóricas, puede notarse que el desarrollo humano y la integralidad de la infancia, se encuentran comprometidos por las formas confusas y dispersas como se está procediendo desde las acciones públicas, ante la detección y activación de rutas de vulneración de derechos. Al respecto, se observan vacíos en el acceso a la Ruta para restablecer derechos, como la desarticulación y/o repetición de acciones, entre los programas e instituciones, que abordan las problemáticas sociales que aquejan la infancia. También, existe una fragmentación de los enfoques y prácticas de carácter protector que se promueven desde las políticas públicas de infancia, lo cual, hace que el desarrollo humano se estanque $y$, en muchos casos, ni siquiera se ocurra.

María José Basoalto (2010) menciona en su investigación que las formas actuales de intervención, tienen como resultado acciones dispersas y descoordinadas al momento de atender a los nińos, asunto que pone en riesgo el cumplimiento del objetivo superior de las políticas públicas que apuntan a la protección y desarrollo integral de la infancia. 


\section{Protección integral}

La protección integral contiene un concepto implícito de justicia, que es responsable de exigir las tareas propias que la garantizan, como resguardar la igualdad de derechos, las libertades de los individuos y buscar el bien de los niños y de la comunidad a la que pertenecen. Esto que indica que, la protección integral alude a la necesidad de un plan de desarrollo que, de manera preventiva responda y garantice el ejercicio pleno de los derechos por parte de los niños, niñas y adolescentes. (Tejeiro, 2005, p.222)

Así las cosas, para la garantía de la protección de los niños, se han definido desde las propuestas públicas, una serie de acciones que van desde la prevención hasta la intervención, sin embargo, -y a pesar de los esfuerzos del programa- estas acciones son poco pertinentes y efectivas. En gran medida, no por la forma como están planteados los lineamientos técnicos, sino por la poca conciencia y compromiso de los funcionarios públicos, en este caso específico, de las agentes educativas.

En muchas ocasiones, en vez de asumir un rol protector, son agentes revictimizantes que rompen las reglas de protección de la información, de oportunidad en la activación de rutas y del acompañamiento a la víctima, lo que coincide con lo expuesto por Jairo García Peña (2016). Este, señala que en el accionar de los funcionarios público no se establece de forma clara el rol que deben desempeñar como agentes garantes de derechos.

Por tanto, los esfuerzos iniciados desde la cotidianidad educativa, para garantizar la atención de la infancia desde distintos niveles, se da de forma desarticulada, tanto entre los programas y las instituciones encargadas de proteger y atender integralmente a los niños, como desde los planes ejecutores de políticas públicas de primera infancia.

Es de resaltar, además, que estas acciones incurren en apuestas asistencialistas, fragmentadas por medio de intervenciones dispersas y poco coordinadas, que no empoderan a las comunidades de sus propios procesos. La vulneración de derechos en la primera infancia está quedando en la mera detección, y pocas veces se garantiza la activación oportuna de una ruta para el inicio de un proceso de restablecimiento de derechos.

\section{Rutas de atención}

Las rutas de atención, como lo define Jairo García Peña (2016), funcionan como guías o caminos a seguir, a partir de la conformación de una red de apoyo institucional que, desde el sector público, pone en acción las competencias institucionales a favor de la atención, prevención, y apoyo integral. A partir de esta red, debe ser posible orientar a las víctimas, acompañantes, funcionarios $y$, en este caso, familias y el agente educativo para el acceso oportuno a los diferentes servicios de atención, en el restablecimiento de derechos.

Pero como se ha expuesto, desde los resultados y los antecedentes, las rutas de atención no son claras para los agentes educativos y personas significativas que se encuentran en el entorno de los niños. Se percibe un notorio desconocimiento de la forma de proceder ante los eventos de violencias sexuales, esto conlleva a que se convierta en una problemática más, además de la que conlleva en si misma el evento violento. Es necesario, en esta vía, preguntarse por los procesos que están entorpeciendo o dificultando la reparación integral, como lo aborda la investigación de María Cristina Sánchez (2008). Esta coincide en plantear que la gravedad de las consecuencias y secuelas resultantes de los abusos sexuales, implica y evidencia la necesidad prevalente de establecer protocolos y rutas claras, que permitan a los diferentes agentes socializados dar respuesta oportuna a las necesidades y situaciones de vulneración a las que se ven expuestos los niños. 
De esta forma, es importante contar con una ruta de atención pertinente que permita abordar y proceder asertivamente en situaciones de vulneración, optimizando tiempos que permitan agilizar la calidad de la atención y evitar la revictimización, asumiendo el rol corresponsable del adulto protector.

\section{Corresponsabilidad}

Como concepto, hace referencia a la concurrencia de actores y acciones encaminadas a la garantía de los derechos de los niños, niñas y adolescentes, función que debe ser asumida tanto por la familia, como por la sociedad y el Estado, en tanto agentes corresponsables de atención, cuidado y protección de los niños, niñas y adolescentes (Hoyos, 2012).

Este proceso de corresponsabilidad, comprende la interiorización del rol del agente educativo, la Escuela, la familia, y la sociedad, como actores a los que les corresponde empoderarse y asumir la protección integral de la infancia, potenciando factores protectores ante la vulnerabilidad.

Como lo sostienen Marcela Sierra, Sara Arboleda, Allison Aguelo (2011) y, Diana Murillo, Julieth Gómez y Lina Quiroz (2011), la sociedad, la familia y el Estado, están llamados a asumir la responsabilidad de la infancia, requiriendo de actuaciones mancomunadas, que permitan el cuidado, atención, protección y bienestar de los niños. Para esto, el Estado requiere de sensibilización y formación en la detección y prevención de las diferentes formas de maltrato infantil, incrementando las habilidades de padres, agentes educativos y personas adultas en general, en la detección y activación oportuna de rutas que permitan restituir los derechos vulnerados de la infancia.

\section{Conclusiones}

Los conocimientos adquiridos por una persona, están conformados por sus experiencias personales y profesionales, y se configuran como la guía del proceder, en este caso de las agentes educativas, al activar rutas que generan o restan efectividad al proceso de restitución de derechos de los niños. Por ello, sería importante promover experiencias formativas de sensibilización dentro de las instituciones de educación inicial que permitan a los profesionales de los equipos interdisciplinarios, la interiorización de aprendizajes significativos con respecto a la vulneración y restitución de derechos.

La cualificación profesional es fundamental para la garantía del desarrollo integral de los niños. La pertinencia en la detección y activación de rutas para el restablecimiento de derechos dependerá de, en gran medida, qué tan preparado se sienta un profesional, de sus recursos disponibles a nivel de conocimiento al momento de realizar el debido proceso. En este sentido, es notorio que la cualificación profesional de los operadores públicos que trabajan con primera infancia, es deficiente, tanto desde la formación como profesionales (académica) hasta la formación recibida en sus lugares de trabajo e institucionalidad pública.

Es importante que se promuevan procesos de formación y cualificación que vayan más allá de la transmisión de información, de jornadas largas de capacitaciones y metodologías poco efectivas; es urgente, pensar en procesos de sensibilización y autorreflexión frente al rol del agente educativo que se compromete a trabajar con la infancia.

Medellín es una ciudad pionera y líder de iniciativas de atención integral a la primera infancia, por medio del programa Buen Comienzo como política pública local, ha logrado la visibilización de los niños, niñas, y familias, como sujetos de derecho desde un enfoque de desarrollo humano integral, sin embargo, es evidente que falta fortalecer la voluntad política de quienes lideran estos procesos. Es evidente que se está fallando en el fortalecimiento de los contextos como potenciadores del desarrollo humano, el cual se ve comprometido por las 
condiciones sociales, familiares, y económicas, que viven las familias vinculadas a este programa.

Para dar respuesta a los requerimientos de atención integral a la primera infancia, es necesario emprender acciones que posibiliten la detección de cualquier tipo de vulneración de derechos y la activación oportuna de rutas, asunto que requiere de acompañamiento, no solo profesional, sino institucional.

La protección integral está ligada a la detección y atención oportuna de las violencias en la niñez, asunto que se atiende desde la activación de rutas; el proceso de denuncia, acompańamiento integral, y restablecimiento, de derechos. Esto depende de las acciones iniciales de activación de ruta, sin embargo, han quedo revelado que no hay unicidad en las maneras de proceder, activar y acompañar a los nińos víctimas y sus familias, lo que conlleva a la revictimización.

Es prioridad, contar con instrumentos, desde el mismo programa, que permita orientar las acciones de detección y atención en casos de vulneración de derechos. También en este sentido, es adecuado invitar a la reflexión sobre formas de construir rutas, desde los equipos interdisciplinarios, procurando contextualizarlas, acorde a las necesidades propias de los contextos y comunidades en las que se atienden los niños.

En este orden de ideas, es necesaria la presencia del Estado, de la familia, sociedad, y de los agentes educativos, como actores primordiales, para la garantía del desarrollo integral de la niñez. Lo anterior, permitirá la detecciòn y atención oportuna de la vulneración de derechos, promoviendo la corresponsabilidad, como principio rector, que orienta las acciones en función de asumir responsabilidad compartida, desde los diferentes roles sociales para proteger y propiciar una vida digna a los niños. Es deber deber del estado, representado en el programa Buen Comienzo, emprender iniciativas más pertinentes, de acompañamiento, formación y generación, de comunidades protectoras de la infancia.

\section{Referencias}

Basoalto, M. (2010). Formas tradicionales de intervención social, fragmentan la integralidad de los niños y las niñas (Tesis de pregrado). Universidad de Chile, Facultad de ciencias sociales Recuperado de http://www.facso.uchile.cl/noticias/81580/ tesinas-de-diplomado-ninez-y-politicas-publicas-en-red-internacional

Bronfenbrenner, U. (1994). Teoría Bioecológica. Traducción Alejandra Devoto. Barcelona: Paidós.

Creswell, J. (2013). Qualitative inquiry research designs: Choosing among five approaches. Los Ángeles: Sage Publications.

Decreto 01277 (2013). Por medio del cual se adoptó la política pública de atención integral a la primera infancia. Gaceta oficial N ${ }^{\circ}$ 4176. Medellín: Alcaldía de Medellín. Recuperado de https://docs.google.com/ file/d/0B8im-ImXZ1KDYlFuZVZmU05iRjQ/view

Deza Villanueva, S. (2005). Factores protectores en la prevención del abuso sexual infantil. Liberabit, 11(11), 19-24. Recuperado de http://www.redalyc. org/pdf/686/68601103.pdf

Dilthey, W. (1990). Die geistige Welt Einleitung in die Philosophie des Lebens. En Gesammelte Schriften, Vol.5. Berlín: Vandenhoeck \& Ruprecht.

García Peña, J. (2016). Propuesta efectiva de ruta de atención intersectorial a las violencias sexuales. Medellín-Colombia: Fondo Editorial Universidad Luis Amigó. Recuperado de http://www.funlam. edu.co/uploads/fondoeditorial/284_Propuesta_ efectiva_de_ruta_de_atencion_intersectorial_a_ las_violencias_sexuales.pdf

Faria, R. y Albuquerque, L. (2008). Professores como Agentes de Prevenção do Abuso Sexual 
Infantil. Educação \& Realidade, 33(2), 209229. Recuperado de http://seer.ufrgs.br/ educacaoerealidade/article/view/7073

Galeano, A. (2012). Sistematización de la práctica profesional en el proceso de aplicación de la ruta de atención a casos de abuso sexual en el centro zonal de Bosa del Instituto Colombiano de Bienestar Familiar (Tesis de pregrado). Corporación Universitaria Minuto de Dios, Facultad de Ciencias Sociales y Humanas, Bogotá, Colombia. Recuperado de http://repository.uniminuto. edu:8080/xmlui/bitstream/handle/10656/1196/ TTS_GaleanoNi\%C3\%B1oAngy_20 12 . pdf?sequence $=1$.

Gómez, E., Salazar, L. \& Zuluaga, C. (2011). Nivel de formación de las docentes y estrategias que utilizan para detectar elabuso sexual en los niñosyniñas en edad preescolar (Tesis de pregrado). Universidad Católica Luis Amigó, Medellín, Colombia. Recuperado de http://www.funlam.edu.co/uploads/facultadeducacion/93_Nivel_de_formaci\%C3\%B3ndedocen. pdf

Gutiérrez, E. (2011). Educadoras de párvulos garantes de derechos de la infancia: ¿un rol sin reflexión? En Osvaldo Torres. Niñez y ciudadanía (108-122). Chile. Dehien Editores.

Hoyos, C. (2012). La corresponsabilidad familiar: Rupturas históricas y prospectiva en Medellín. Colombia: Unaula.

Jiménez, X. y Giraldo, L. (2011). Caracterización de los presuntos delitos sexuales en la ciudad de Medellín en 2010. Revista de la Facultad de Ciencias Forenses de la Salud C.F.S., (7) 75-90. Recuperado de http://ojs.tdea.edu.co/index.php/forenses/ article/view/84.

Lefio, L., Silva, h. y Rivas, K. (2013). Maltrato infantil en la primera infancia: una revisión panorámica sobre prevención, detección y tratamiento. Medwave, 13(7), 1-9. Recuperado de http://files. sld.cu/renacip/files/2014/06/maltrato-infantil.pdf doi: $10.5867 /$ medwave.2013.07.5748

Ley 1098 de 2006 [Congreso de la República de Colombia]. Por medio del cual se expide el Código de Infancia y Adolescencia en Colombia. Recuperado de http://slideshare.net/freddymon/ ley-1098de-2006-codigo-de-la-infancia-y-la-adolescencia

Ley 1146 de 2007 [Congreso de la República de Colombia]. Por medio de la cual se expiden las normas para la prevención de la violencia sexual y atención integral de los nińos, niñas y adolescentes abusados sexualmente. Colombia. Recuperado de http://www.mineducacion.gov.co/normatividad/1753/articles-146167_archivo_pdf.unknown/

Lineamientos y estándares para la prestación del servicio de atención integral a la primera infancia. (2015). Alcaldía de Medellín. Programa Buen Comienzo. Medellín.

López, J. (2001). Investigación cualitativa y participativa: Un enfoque histórico-hermenéutico y crítico-social en Psicología y educación ambiental. Medellín: Universidad Pontificia Bolivariana.

Max-Neef, A. (1994). Desarrollo a Escala Humana. Barcelona: Icaria Editorial

Morillo, B., Montero, L.y Colmenares, Z. (2012). Conocimiento de los padres en la prevención del abuso sexual infantil. Enfermería Global, 11(25), 1-7, doi: https://dx.doi.org/10.4321/ S1695-61412012000100001

Mouesca, J. (2015). Prevención del maltrato infantil: función del pediatra. 2da parte. Prevención antes de que ocurra, ante la sospecha y con la confirmación del maltrato. Archivos argentinos de pediatría, 114(1), 64-74, doi: https://dx.doi.org/ http://dx.doi.org/10.5546/aap.2016.64

Murillo, D., Gómez. J. y Quiroz, L. (2011). Prevención contra la violencia y la protección a la 
primera infancia, un asunto de todos (Tesis de pregrado). Universidad San Buenaventura, Medellín, Colombia. Recuperado de http://bibliotecadigital.usbcali.edu.co/jspui/bitstream/10819/846/1/ Prevencion_Violencia_Proteción_Murillo_2011.pdf

NavasTremont, G. (2011). Formación docente en el área de la sexualidad para preescolar. Revista de Investigación, 35(74), 89-109. Recuperadodehttp:// nnn.redalyc.org/articulo.oa?id=376140389005

Olaz, A. (2011). Una aproximación conceptual a la cualificación profesional desde una perspectiva competencial. Papers Revista Sociológica. 96(2), 589-616, doi: http://dx.doi.org/10.5565/rev/ papers/v96n2.154

Pardo, M. (2009). Sistematización de la experiencia en trabajo social frente a la atención integral a los casos de abuso sexual infantil desde la mirada de redes sociales en la comisaría de Soacha Cundinamarca (Tesis de pregrado). Corporación Universitaria Minuto de Dios, Facultad de Ciencias Humanas y Sociales, Bogotá, Colombia. Recuperado de http://repository. uniminuto.edu:8080/jspui/bitstream/10656/782/1/ TTS_PardoArdilaMariedDayan_2009.pdf.

Peroni, G. \& Prato, J. (2012). Aportes para la intervención en maltrato y abuso sexual infantil y adolescente. Uruguay: UNICEF

Ramírez, V., Aranceta, M. y Montoya, F. (2015). Contradicciones en el proceso de restablecimiento de derechos de los niños, niñas y adolescentes en Colombia (Tesis de especialización). Universidad de Medellín, Colombia. Recuperado de http://repository.udem.edu.co/bitstream/handle/11407/1219/ Contradicciones.pdf?sequence $=1$ \&isAllowed $=y$

Rodríguez Cely, L A; (2003). Intervención interdisciplinaria en casos de abuso sexual infantil. Universitas Psychologica, 2. 57-60. Recuperado de http://www.redalyc.org/articulo.oa?id=64720108

Sánchez, M. (2008). Caracterización psicopatológica de niños, niñas y adolescentes víctimas de abuso sexual, ubicados bajo medidas de protección en hogares sustitutos del ICBF en la ciudad de Bucaramanga y adecuación del modelo de atención a través de la creación de un protocolo de actuación frente al abuso sexual: una experiencia piloto (Tesis de especialización). Universidad Pontificia Bolivariana, Bucaramanga, Colombia. Recuperado de https://repository.upb. edu.co/bitstream/handle/20.500.11912/244/digital_16640.pdf?sequence $=1$ \&isAllowed $=y$

Sen, A. (2000). Desarrollo y Libertad. Buenos Aires: Planeta.

Sierra Toro., M. Arboleda Londońo, S. y Agudelo Obando, A. (2011). Construyendo indicadores de protección para la primera infancia, una tarea de corresponsabilidad (Tesis de pregrado). Universidad de San Buenaventura, Medellín, Colombia. Recuperado de http://bibliotecadigital.usbcali.edu. co:8080/bitstream/10819/847/1/Construyendo_ Indicadores_Protecci\%C3\%B3n_Sierra_2011.pdf

Tamir, P. (2005). Conocimiento profesional y personal de los profesores y de los formadores de profesores. Currículum y formación del profesorado. 9(2). Granada. Recuperado de https://www.ugr. es/ -recfpro/rev92ART3.pdf

Tejeiro, C. (2005). Teoría general de la niñez y adolescencia. Segunda edición. Bogotá: Uniandes.

Villegas, M., Zapata, I. \& Vega, L. (2011). Seguimiento, monitoreo y evaluación a la protección de la primera infancia desde las comunidades (Tesis de pregrado). Universidad San Buenaventura, Medellín, Colombia. Recuperado de http://bibliotecadigital.usbcali.edu.co/jspui/ bitstream/10819/849/1/Seguimiento_Monitoreo Evaluacion_Vega_2011.pdf

Zapata, B. y Ceballos, L; (2010). Opinión sobre el rol y perfil del educador para la primera infancia. Revista Latinoamericana de Ciencias Sociales, Niñez y Juventud, 8. 1069-1082. Recuperado de http:// www.redalyc.org/articulo.oa?id $=77315155021$ 


\section{Datos de filiación}

John Jairo García Peńa. Psicólogo, especialista en psicoanálisis con niños, y Magister en Desarrollo. Docente Universidad Católica Luis Amigó y Universidad de Antioquia. Ha realizado proyectos en el sector público, Medellín, Colombia. Sus líneas de Investigación fundamentales son Desarrollo Humano y violencias sexuales, sobre las que ha contribuido en varios libros y artículos.

Correo electrónico: carolinasegura2000@gmail.com
Carolina Restrepo Segura. Psicóloga, con Master en Intervenciones psicosociales, y se desempeña como Psicóloga de la Colegiatura colombiana, como líder de los procesos de Bienestar universitario. Sus líneas de investigación más importantes es el estudio de las formas de violencias sexuales.

Correo electrónico: john.garciape@amigo.edu.co 